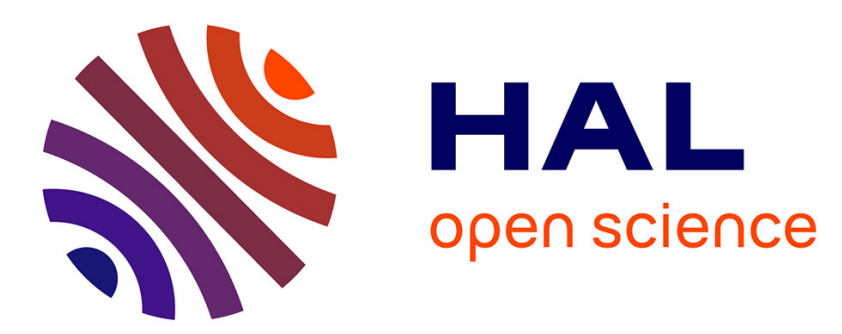

\title{
The significance of local thermal non-equilibrium in simulations of enhanced geothermal recovery
}

\author{
Rachel Gelet, B Loret, N Khalili
}

\section{To cite this version:}

Rachel Gelet, B Loret, N Khalili. The significance of local thermal non-equilibrium in simulations of enhanced geothermal recovery. 14thIACMAG, Sep 2014, Kyoto, Japan. pp.1713 - 1718, 10.1201/b17435-303 . hal-01079193

\section{HAL Id: hal-01079193 \\ https://hal.science/hal-01079193}

Submitted on 31 Oct 2014

HAL is a multi-disciplinary open access archive for the deposit and dissemination of scientific research documents, whether they are published or not. The documents may come from teaching and research institutions in France or abroad, or from public or private research centers.
L'archive ouverte pluridisciplinaire HAL, est destinée au dépôt et à la diffusion de documents scientifiques de niveau recherche, publiés ou non, émanant des établissements d'enseignement et de recherche français ou étrangers, des laboratoires publics ou privés. 


\title{
The significance of local thermal non-equilibrium in simulations of enhanced geothermal recovery
}

\author{
R.M. Gelet \\ GeM Laboratory, University of Nantes, ECN, CNRS, B.P. 92101, 44321 Nantes, Cedex 3, France.
}

B. Loret

Laboratoire Sols, Solides, Structures, Institut National Polytechnique de Grenoble, B.P. 53X, 38041 Grenoble Cedex, France.

N. Khalili

School of Civil and Environmental Engineering, The University of New South Wales, Sydney 2052, Australia.

\begin{abstract}
Accessing the thermo-mechanical response of large deep hot dry rock (HDR) reservoirs during geothermal extraction remains a challenging task that can be comprehended with numerical tools. Of crucial importance to the economic viability of these HDR reservoirs is the knowledge of thermal output evolution, fluid excessive pressure and induced thermal stress, at various steps of the circulation tests. Thermal recovery from a HDR reservoir, viewed as a deformable fractured medium, is investigated with a focus on the assumption of local thermal non-equilibrium (LTNE). To this end, a fully coupled finite element formulation for a thermo-elastic fractured medium in LTNE is developed (Gelet et al. 2013). Hydraulic diffusion, thermal diffusion, forced convection and deformation are considered in a two-phase framework, the solid phase being made by impermeable solid blocks separated by saturated fractures. Each of the two phases is endowed with its own temperature. The resulting system of equations is used to address a generic HDR reservoir subjected to temperature and pressure gradients. A change of time profile of the outlet fluid temperature is observed as the fracture spacing increases, switching from a single-step pattern to a double-step pattern, a feature which is viewed as characteristic of established LTNE. A dimensionless number is proposed to delineate between local thermal equilibrium (LTE) and non-equilibrium. This number embodies local physical properties of the mixture, elements of the geometry of the reservoir and the production flow rate. All the above properties being fixed, the resulting fracture spacing threshold between LTNE and LTE is found to decrease with increasing porosity. The thermally induced effective stress is tensile near the injection well, illustrating the thermal contraction of the rock, while the pressure contribution of the fracture fluid is negligible during the late period.
\end{abstract}

\section{INTRODUCTION}

The production of geothermal energy is achieved by cold water injection, in fractured igneous rocks (originally with low matrix permeability), at sites where the vertical temperature gradient is favorable. Of crucial importance to the economic viability of these HDR reservoirs is the knowledge of thermal output evolution, induced thermal stress and fluid loss, at various time scales of the circulation tests (Armstead \& Tester 1987). A key factor influencing geothermal energy recovery is the difference in characteristic times between diffusion in the solid phase and convection in the fluid phase. The thermally induced effective stress which results from these two contributions may lead to permeability change and fluid loss. Also, thermal shrinkage and pressure drop, across the body of the reservoir, occur at various time scales and the understanding of their interaction requires the simulation of a continuum mixture in LTNE (Aifantis 1980).

Coupled thermo-hydro-mechanical analyses for geothermal systems are scarce (Hayashi et al. 1999, O'Sullivan et al. 2001) and focus mainly on partially coupled systems in an effort to implement a precise fracture network through a system of discrete discontinuities (DuTeaux et al. 1996, Bruel 2002), to couple free and forced convection (Bataillé et al. 2006), or to characterize joint closure with a stress dependent law (Kohl \& Hopkirk 1995, Bower \& Zyvoloski 1997) 
or stress-dissolution/precipitation effects (Taron et al. 2009). However, before Gelet (2011) no numerical analysis was proposed to simulate reservoir behavior with a continuum mixture in LTNE.

Extending previous studies, Loret \& Khalili (2000) and Khalili \& Loret (2001) proposed comprehensive fully coupled thermo-hydro-mechanical formulations based on the mixture theory for unsaturated porous media. Both contributions focus on the importance of an appropriate definition of the effective stress. In the present contribution, the work of Khalili \& Loret (2001) is extended to describe thermal recovery from a geothermal reservoir viewed as a deformable fractured medium in LTNE (Gelet et al. 2013).

\section{THERMOPOROELASTIC MODEL IN LTNE}

Hydraulic diffusion, thermal diffusion, forced convection and deformation are considered in a twophase framework, the solid phase being made by impermeable solid blocks separated by saturated fractures. Local thermal non-equilibrium between the phases holds at all points of the simulated zone. Each phase $k$ is endowed with its own temperature $T_{k}$ and its own material properties. LTNE emanates from the contrast between the rapid convection of heat by the moving fluid in the fractures and the slow diffusion of heat through the solid matrix. Indeed, the characteristic time associated with convection is several orders of magnitude smaller than the characteristic times of diffusion in both solid skeleton and fluid.

\subsection{Governing equations}

Within a single porosity conceptual framework (Gelet et al. 2013), differential equations describing the problem at hand may be expressed as,

$$
\begin{gathered}
\mu^{\mathrm{DS}} \nabla^{2} \mathbf{u}+\left(\lambda^{\mathrm{DS}}+\mu^{\mathrm{DS}}\right) \nabla(\operatorname{div} \mathbf{u}) \\
-\xi_{f} \nabla p_{f}-\frac{c_{T}}{c} \nabla T_{s}+\rho \mathbf{g}=\mathbf{0} \\
-\operatorname{div}\left(\frac{k_{f}}{\mu_{f}}\left(\nabla p_{f}-\rho_{f} \mathbf{g}\right)\right)+a_{f f} \frac{\partial p_{f}}{\partial t} \\
+\xi_{f} \operatorname{div} \frac{\partial \mathbf{u}}{\partial t}+a_{f T_{s}} \frac{\partial T_{s}}{\partial t}+a_{f T_{f}} \frac{\partial T_{f}}{\partial t}=0
\end{gathered}
$$

$$
\begin{array}{r}
-\operatorname{div}\left(n_{s} \Lambda_{s} \nabla T_{s}\right)+a_{T_{s} T_{s}} \frac{\partial T_{s}}{\partial t} \\
+T_{s} a_{f T_{s}} \frac{\partial p_{f}}{\partial t}+T_{s} \frac{c_{T}}{c} \operatorname{div} \frac{\partial \mathbf{u}}{\partial t} \\
+\kappa_{s f}\left(T_{s}-T_{f}\right)=0
\end{array}
$$

$$
\begin{array}{r}
-\operatorname{div}\left(n_{f} \Lambda_{f} \nabla T_{f}\right)+a_{T_{f} T_{f}} \frac{\partial T_{f}}{\partial t} \\
+T_{f} a_{f T_{f}} \frac{\partial p_{f}}{\partial t}+\rho_{f} \mathbf{J}_{f} \cdot \nabla H_{f} \\
+\kappa_{s f}\left(T_{f}-T_{s}\right)=0
\end{array}
$$

in which

$$
\begin{aligned}
& \xi_{f}=1-c_{s} / c \\
& a_{f f}=n_{f} c_{f H}+\left(\xi_{f}-n_{f}\right) c_{s} \\
& a_{f T_{s}}=\left(n_{f}-\xi_{f}\right) c_{T} \\
& a_{f T_{f}}=-n_{f} c_{f T} \\
& a_{T_{s} T_{s}}=n_{s} \rho_{s} C_{s}^{(v)} \\
& a_{T_{f} T_{f}}=n_{f} \rho_{f} C_{f}^{(p)} \\
& \nabla H_{f}=\left(1-T_{f} c_{f T}\right) \frac{\nabla p_{f}}{\rho_{f}}+C_{f}^{(p)} \nabla T_{f} .
\end{aligned}
$$

where $\mathbf{u}$ is the displacement of the solid, $p_{f}$ is the pressure of the fracture fluid, $T_{s}$ is the temperature of the solid and $T_{f}$ that of the fracture fluid. Throughout, the indices $s$ and $f$ refer to the solid and to the fluid, respectively.

Equation (1) uses the Lamé constants of the drained solid $\lambda^{\mathrm{DS}}$ and $\mu^{\mathrm{DS}}$, the body force vector $\mathbf{g}$, the overall density $\rho$, the compressibility of the mixture $c$, the compressibility of the solid grains $c_{s}$, and the volumetric thermal expansion coefficient of the solid phase $c_{T}$.

Equation (2) requires the macroscopic porosity $n_{f}$, the macroscopic intrinsic permeability $k_{f}$, and the dynamic viscosity $\mu_{f}$. The hydraulic compressibility $c_{f H}$ and the thermal compressibility $c_{f T}$ are defined from the intrinsic density $\rho_{f}$ of the fluid.

Equation (3) involves the solid heat capacity at constant volume and fluid pressure $C_{s}^{(v)}$, the solid density $\rho_{s}$ and porosity $n_{s}$, and the solid thermal conductivity $\Lambda_{s}$. The specific inter-phase heat transfer parameter parameter $\kappa_{s f}$ is discussed in Section 2.2.

Finally, equation (4) involves the fluid heat capacity at constant fluid pressure $C_{f}^{(p)}$, the apparent volume flux $\mathbf{J}_{f}$ (Darcy's law), and the fluid thermal conductivity $\Lambda_{f}$.

Equations (1) to (4) are direct consequences of the field equations (balance of momentum for the mixture, balance of mass for the fluid, balance of energy for the solid and balance of energy for the fluid). Additional information on the parameters of the model can be found in previous works Gelet et al.(2011, 2013).

The approach is amendable to a double porosity framework: it can be extended to zoom on the solid blocks and include their innate pore pressure, temperature and the fluid and energy exchanges with the fractures (Gelet et al. 2012). 
The weak form of the governing equations, the discretization and time-integration procedures to solve the coupled equations through a finite element method are detailed in Gelet (2011). The primary variables are the displacement vector, the pressure of the fluid, the temperature of the solid and the temperature of the fluid. The resulting system of equations is used to address a generic HDR reservoir subjected to temperature and pressure gradients.

\subsection{The specific inter-phase heat transfer parameter $\kappa_{s f}$}

The coefficient of specific inter-phase heat transfer $\kappa_{s f}\left[\mathrm{~W} / \mathrm{m}^{3} . \mathrm{K}\right]$ controls the rate at which the two phase system (solid-fluid) reaches thermal equilibrium. The higher this coefficient, the faster thermal equilibrium is reached. This coefficient can be defined by idealizing the solid blocks to squares of length $B$ bordered by fractures of width $2 b$ (Fig. 1).

In geothermal applications, the fracture width is much smaller than the fracture spacing, namely $2 b \ll$ $B$. Hence, the specific coefficient of heat transfer between the solid and the fluid phase can be linearly related to $B^{-2}$ (Gelet et al. 2013),

$\kappa_{s f}=\frac{8}{B^{2}} \frac{n_{s} \Lambda_{s} \times 2 \Lambda_{f}}{n_{s} \Lambda_{s}+2 \Lambda_{f}}$.

The thermal conductivities of the two phases are involved. For a geothermal reservoir, typical values entail $n_{s} \approx 1, \Lambda_{s}=2.71 \mathrm{~W} / \mathrm{m} . \mathrm{K}$ and $\Lambda_{f}=0.6 \mathrm{~W} / \mathrm{m} . \mathrm{K}$, and the two phases are seen to contribute to the overall conductivity.

Since $\kappa_{s f}$ depends on the solid-fluid specific surface, the experimental measurement of this coefficient proves to be difficult. One way around is to calibrate the specific inter-phase heat transfer coefficient, with help of data provided from a specific HDR reservoir as proposed in Gelet et al. (2012, 2013). Instead, here, a sensitivity analysis is performed to determine the threshold value that separates local thermal equilibrium from the local thermal non-equilibrium.

\section{HDR RESERVOIR ANALYSIS}

The fully coupled thermo-hydro-mechanical model is used in the subsequent section to investigate the thermal drawdown of a generic HDR reservoir in LTNE. Information on the geometry and the boundary conditions are provided first.

\subsection{Geometry}

An artificially fractured reservoir with horizontal injection and production wells is considered (Fig. 1). The simulations assume a plane strain analysis, in the $x-z$ plane, and symmetry with respect to $z$-axis. The finite element mesh includes half of the reservoir and

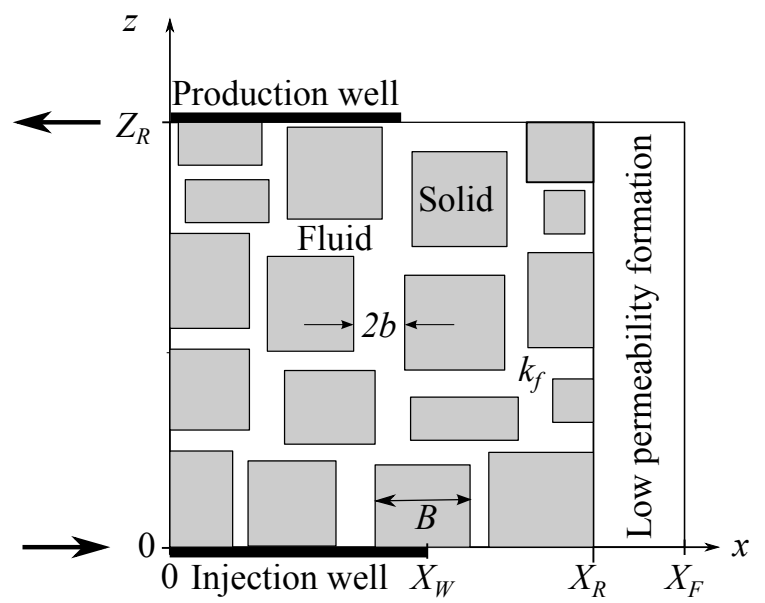

Figure 1: Representation of a generic HDR reservoir (not at scale). The permeability $k_{f}$ of the reservoir depends on the average fracture spacing $B$ and on the average fracture aperture $2 b$.

a portion of the surrounding low permeability rock formation. The HDR fractured reservoir is idealized by a single porosity saturated medium.

The injection and the production wells are located at the bottom and at the top of the reservoir, respectively. The length of the wells is a key parameter that governs the flow path and the heat transfer between the rock and the fluid. Here, the wells are assumed to penetrate horizontally into the entire reservoir, that is $X_{W}=X_{R}$, and the velocity of the fluid $\mathbf{v}_{f}$ is vertical and uniform between the two wells. The main purpose of this paper is to focus on the influence of LTNE and alternative setups (flow paths) are described elsewhere (Gelet et al. 2013).

\subsection{Boundary conditions}

The thermal, hydraulic and mechanical boundary conditions are sketched in Fig. 2.

The injection temperature $T_{\text {inj }}$ is applied to all the phases along the injection well. The outlet temperature $T_{f, \text { out }}$ along the producing well is an output of the simulations. The remaining horizontal and vertical boundaries are thermally insulated from the surroundings.

The injection and production pressures, $p_{f, \text { inj }}$ and $p_{f, \text { out }}$ respectively, are both specified along the injection and production wells. The remaining boundaries are hydraulically impermeable.

The vertical stress $\sigma_{\mathrm{v}}$ remains constant along the top boundary $z=Z_{R}$, and so does the horizontal stress $\sigma_{\mathrm{H}}$ along the lateral boundary $x=X_{F}$. The displacements on the other boundaries are specified, $u_{x}(x=0, z)=0$ and $u_{z}(x, z=0)=0$.

At the contact between the reservoir and the surrounding rock $x=X_{R}$, the reservoir is assumed to be thermally insulated from the rock formation. Alternative setups are studied in Gelet et al. (2013). 

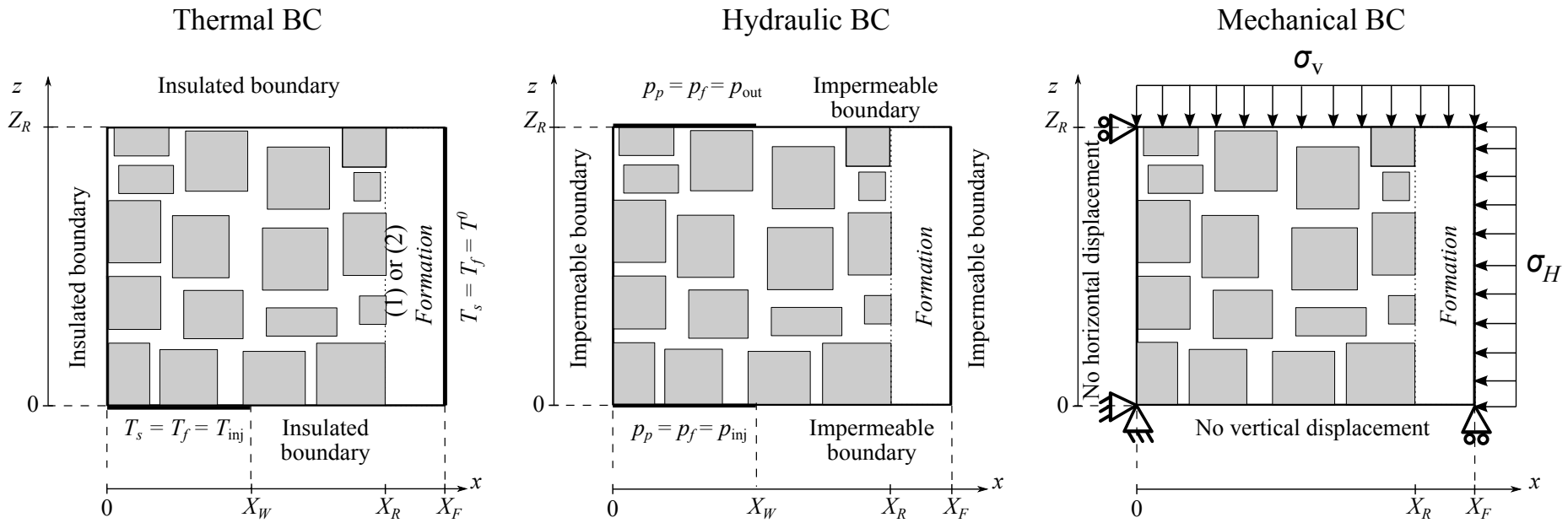

Figure 2: Thermal, hydraulic and mechanical boundary conditions.

\section{NUMERICAL RESULTS}

The resulting system of equations is used to address a generic HDR reservoir subjected to temperature and pressure gradients with a focus on the LTNE assumption. First, thermal drawdown curves (time profiles of the fluid temperature at the producing well) are observed to show a single-step pattern for small fracture spacings $B$ and double-step patterns for large fracture spacings (Sect. 4.1). The threshold values between LTNE and LTE are sought in terms of the fracture spacing $B_{T}$ for an average steady state velocity $\mathrm{v}^{\infty}$ and several fluid porosities in Sect.4.2. Next, a dimensionless parameter is proposed in Sect. 4.3 to delineate between local thermal equilibrium (LTE) and non-equilibrium. Finally, the reservoir response in terms of overall effective stress is presented in Sect. 4.4.

Throughout, temperatures are presented in a dimensionless format. The dimensionless fluid temperature $T_{D}$ built from the outlet fluid temperature $T_{f \text {,out }}$, the initial temperature $T^{0}$ and the injection temperature $T_{\mathrm{inj}}$,

$T_{D}=\frac{T^{0}-T_{f, \text { out }}}{T^{0}-T_{\text {inj }}}$,

ranges between $0\left(T_{f, \text { out }}=T^{0}\right)$ at early time and 1 $\left(T_{f, \text { out }}=T_{\text {inj }}\right)$ ultimately.

\subsection{The double step pattern}

A change of time profile is observed as the fracture spacing $B$ increases (Fig. 3, center). Time profiles for small $B$ are clearly dominated by convection and display a characteristic single-step pattern. Conversely, the time profiles for large $B$ display three stages and typical double-step patterns.

(1) In the first stage, the strong increase of the dimensionless temperature corresponds to the abrupt propagation of the injection temperature dominated by convection. This effect is mostly attributed to the difference in characteristic times between forced convection in the fracture network and diffusion of heat in the solid matrix. Accordingly for each fracture porosity $n_{f}$, the magnitude of the dimensionless temperature $T_{D}$ at the end of this first stage is increasing as a function of $B$. Thus, the smaller $B$ (the larger the specific solid-fluid heat transfer $\kappa_{s f}$ ), the higher the 'instantaneous damping' of the cooled fluid front by the solid phase. On the other hand, the duration of the first stage is independent of $B$, and it is about equal to the characteristic time for a particle of fluid to flow the length $Z_{R}$ at the steady velocity $\mathrm{v}^{\infty}$,

$t_{c}^{\text {stage } 1}=\frac{Z_{R}}{\mathrm{~V}^{\infty}} \approx 2 \times \tau_{S 1}=13$ days.

(2) During the second stage, the dimensionless temperature remains constant and displays a plateau. Heat transfer between the solid and the fluid phases takes place partly at constant fluid temperature.

(3) The third stage is characterized by a second strong increase of the temperature $T_{D}$. The time required for the outlet temperature $T_{f \text {,out }}$ to reach $95 \%$ of the injection temperature $T_{\mathrm{inj}}$ is higher for a model in LTNE than in LTE. In other words, the response of the mixture is delayed by the transfer of heat from the solid phase to the fluid phase. This type of behavior is characteristic of the response of phases in nonequilibrium: as an example, the consolidation time of a dual porosity medium is delayed compared with a single porosity medium (Khalili et al. 1999).

\subsection{Influence of the porosity $n_{f}$}

The influence of the fracture porosity, in the range of $0.001<n_{f}<0.01$, is mainly visible during the first and the second stage of the double-step pattern (Fig. 3). (1) For each fracture spacing $B$, the magnitude of $T_{D}$ at the end of this first stage is increasing as a function of the fluid volume ratio $n_{f}$, i.e. the larger the volume of cooled fluid, the smaller the temperature outlet. Since $n_{s} \approx 1$, the rate of heat transfer can be considered as constant, eqn (6), as opposed to the overall amount of heat supply in the system. In addition, the duration of the first stage is independent of $n_{f}$ in agreement with eqn (8). 

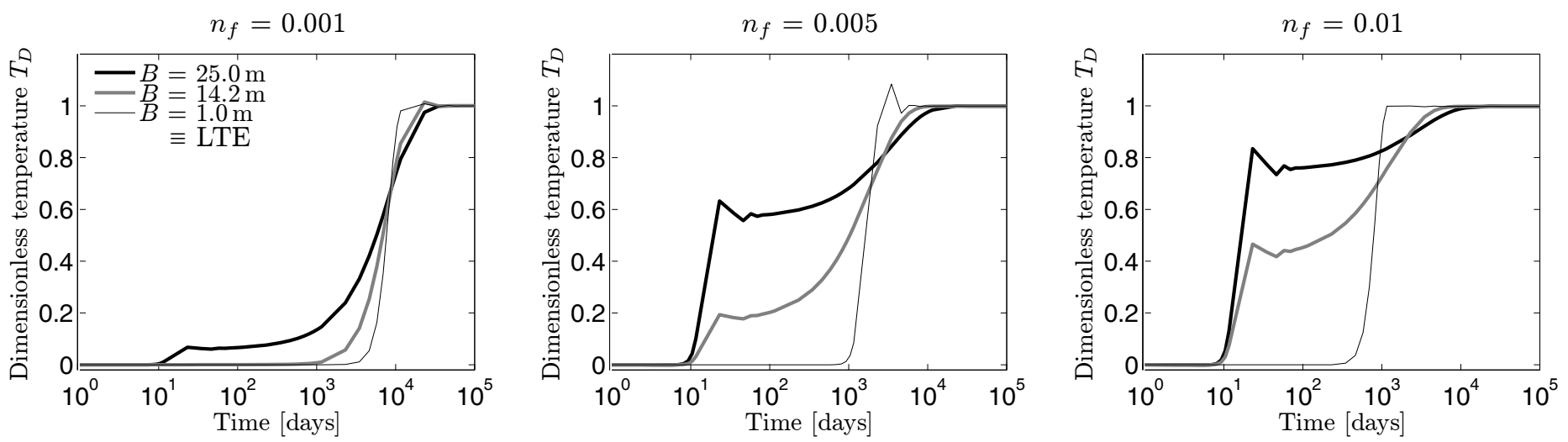

Figure 3: Dimensionless temperature outlet $T_{D}$, eq. (7), as a function of time for three porosities $n_{f}$ and three fracture spacings $B$. All results are for $Z_{R}=230 \mathrm{~m}, \mathrm{v}^{\infty}=2 \times 10^{-4} \mathrm{~m} / \mathrm{s}$ and thermal properties from Gelet et al. (2013). In LTNE, time profiles display a double-step pattern, whereas in LTE, time profiles display a continuous pattern. The thresholds between LTE and LTNE are associated with the fracture spacings $B_{T}=6 \mathrm{~m}$ (left), $B_{T}=2.5 \mathrm{~m}$ (middle) and $B_{T}=2 \mathrm{~m}$ (right). The corresponding dimensionless values of $\eta_{D}$ are equal respectively to 11,13 and 10 . Therefore, LTE is associated with $\eta_{D}$ larger than, say 13 , while values of $\eta_{D}$ smaller than 13 require a LTNE analysis.
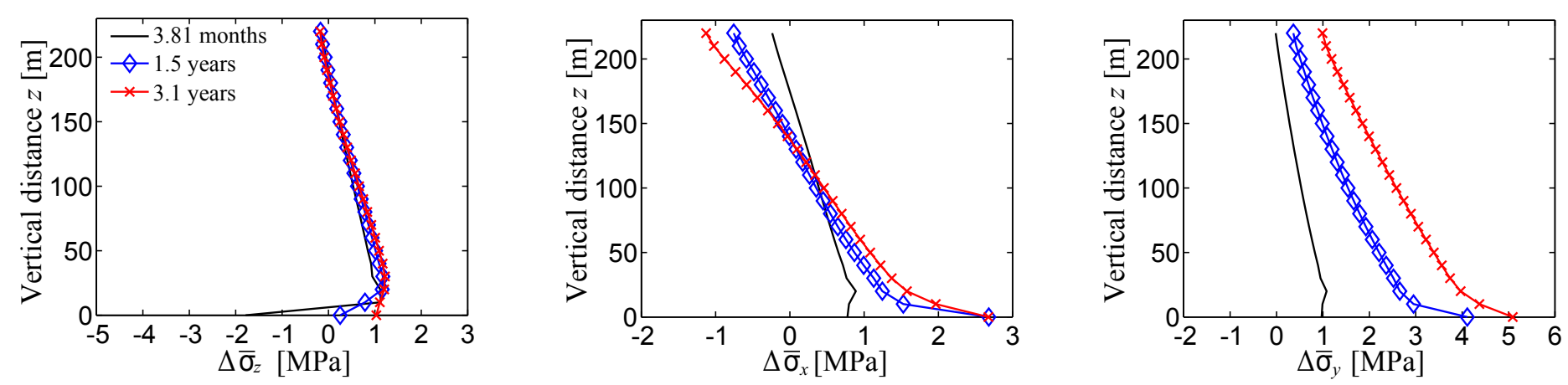

Figure 4: Late period profiles, along the $z$-axis at $x=60 \mathrm{~m}$, of the induced change in vertical effective stress (left), lateral effective stress (center) and out of plane effective stress (right), with $n_{f}=0.005, k_{f}=8.0 \times 10^{-15} \mathrm{~m}^{2}, \kappa_{s f}=33.0 \mathrm{~mW} / \mathrm{m}^{3} . \mathrm{K}$. Tensile stresses are counted positive. The contraction of the solid phase induces the effective lateral stress to be tensile near the injection well $(z<$ $120 \mathrm{~m})$ and compressive near the production well $(z>120 \mathrm{~m})$.

(2) Time profiles in Fig. 3 indicate that increasing the porosity of the fracture network $n_{f}$ reduces the time length of the second stage and, hence, speeds up thermal depletion. An increase of fluid porosity modifies the phase and the heat partition in the system and hence reduces the overall amount of heat to be transferred by the solid to the fluid. Since the rate of heat transfer is almost independent of the fluid porosity, see eqn (6) in which $n_{s} \approx 1$, the heat transfer period requires less time.

In terms of fracture spacing, the threshold between LTE and LTNE decreases with increasing fluid porosity, $B_{T}=6 \mathrm{~m}\left(n_{f}=0.001\right), B_{T}=2.5 \mathrm{~m}\left(n_{f}=0.005\right)$ and $B_{T}=2 \mathrm{~m}\left(n_{f}=0.01\right)$, due to the increasing amount of cooled fluid. Those values are obtained by trial and error and correspond to $T_{s}=T_{f}$, at the reservoir outlet, over the entire circulation test. For smaller fracture spacings $B<B_{T}$, the local thermal non-equilibrium responses are indistinguishable from the responses in equilibrium.

\subsection{A dimensionless LTNE parameter}

In the context of geothermal energy recovery, reservoir performances can be expressed in terms of a dimensionless LTNE parameter (Gringarten et al.
1975). The dimensionless LTNE parameter $\eta_{D}$ serves to delineate LTE and LTNE and reflects the overwhelming importance of the characteristic lengths of the fracture network, namely the average aperture $2 b$ and the average spacing $B$.

The general form of $\eta_{D}$ (Gelet et al. 2013) expresses in terms of the thermal properties of the porous medium $\left(\Lambda_{s}, \Lambda_{f}\right.$ and $\left.C_{f}^{(p)}\right)$, the fluid porosity $n_{f}$, the diffusion-convection length $Z_{R}$, the fracture spacing $B$, and the steady state fluid velocity $\mathrm{v}^{\infty}$. For $n_{s} \Lambda_{s} \gg n_{f} \Lambda_{f}$ and for $n_{s} \approx 1$, the dimensionless LTNE parameter writes

$\eta_{D}=\frac{1}{n_{f}} \frac{16 \Lambda_{f}}{\Lambda_{s}+2 \Lambda_{f}} \frac{Z_{R} \alpha_{T, f}}{\mathrm{v}^{\infty} B^{2}}$.

in which $\alpha_{T, f}=n_{f} \Lambda_{f} / \rho_{f} C_{f}^{(p)}$ is the thermal diffusivity of the fluid and $B$ depends on the parameters $n_{f}$ and $2 b, B=4 b / n_{f}$, for idealized solid square blocks of length $B$ borded by fractures of width $2 b$ (Sect. 2.2 and Fig. 1).

In the previous section, three fracture spacing thresholds $B_{T}$ have been identified, one for each porosity. The corresponding dimensionless values of $\eta_{D}$ are equal respectively to $11\left(n_{f}=0.001\right), 13\left(n_{f}=\right.$ $0.005)$ and $10\left(n_{f}=0.01\right)$. Consequently, within the range of interest $0.001 \leq n_{f} \leq 0.01$, dimensionless 
values $\eta_{D} \geq 13$ are likely to represent LTE and $\eta_{D}<$ 13 represents LTNE.

\subsection{THM coupled behavior}

The LTNE model is used to investigate the coupled thermo-hydro-mechanical behavior of the Fenton Hill HDR reservoir (Fig. 4).

The coupled behavior of porous media in LTNE is governed by the difference in characteristic times between the thermal depletion of the fluid phase and that of the solid phase. During the first weeks, the thermal depletion of the fluid has already started to propagate inside the reservoir, whereas the thermal depletion of the solid is confined to the immediate vicinity of the injection well. However, as heat transfer between the two constituents gets completed, the mixture tends to thermal equilibrium. On the other hand, the pressure field reaches steady state within a few hours due to the high hydraulic diffusivity of the fracture network. The large difference between the characteristic times of the fracture fluid response (early/intermediate period) and of the solid temperature response (late period) allows segregation of the respective contributions to the effective stress due to hydraulic and thermal effects. As expected, the latter is larger than the former especially in the horizontal and out of plane directions. The effective stresses are governed by the thermal depletion of the solid phase during the late period. Cooling of the lower part of the reservoir induces characteristic contractive strains and tensile stresses.

\section{CONCLUSIONS}

Diffusion and forced convection mechanisms between two phases have been studied in the context of heat extraction from a fractured hot dry rock (HDR) reservoir. A model describing the behavior of poroelastic fractured media has been extended to account for local thermal non-equilibrium (LTNE).

A parametric analysis has been carried out to study the influences of the solid-fluid heat transfer coefficient and of the fluid porosity. As a typical feature of LTNE, the temperature outlet profile displays a double-step pattern, which is interpreted as a characteristic of established LTNE. An attempt to define a dimensionless threshold above which a LTNE analysis is required is exposed (Sect. 4.3).

The model is used to describe the thermo-hydromechanical behavior of a long term circulation test on the Fenton Hill HDR reservoir. It has been found that the thermally induced effective stresses are tensile near the injection well due to the thermal contraction of the solid, suggesting a possible increase of fracture aperture. This work highlights how LTNE versus LTE can strongly impact the mechanical response of the reservoir and possibly affect the evolution of the fracture network. The LTNE model leads to a characteristic double-step reservoir response.

\section{REFERENCES}

Aifantis, E. C. (1980). Further comments on the problem of heat extraction from hot dry rocks. Mechanics of Research Communications 7 (4), 219-226.

Armstead, H. \& J. Tester (1987). Heat mining. E.\&F.N. Spon Ltd., London and New York.

Bataillé, A., P. Genthon, M. Rabinowicz, \& B. Fritz (2006). Modeling the coupling between free and forced convection in a vertical permeable slot: implications for the heat production of an enhanced geothermal system. Geothermics 35, 654-682.

Bower, K. \& G. Zyvoloski (1997). A numerical model for thermo-hydro-mechanical coupling in fractured rock. International Journal of Rock Mechanics and Mining Sciences 34, 1201-1211.

Bruel, D. (2002). Impact of induced thermal stresses during circulation tests in an engineered fractured geothermal reservoir: Example of the Soultz-Sous-Forêts european hot fractured rock geothermal project, rhine graben, france. Oil \& Gas Science and Technology 57, 459-470.

DuTeaux, R., D. Swenson, \& B. Hardeman (1996). Insight from modelling discrete fractures using GEOCRACK. In Proceedings, Twenty-First Workshop on Geothermal Reservoir Engineering Stanford University, Stanford. California, pp. 287293.

Gelet, R. (2011). Thermo-hydro-mechanical study of deformable porous media with double porosity in local thermal nonequilibrium. Ph. D. thesis, Institut National Polytechnique de Grenoble, France, and The University of New South Wales, Sydney, Australia.

Gelet, R., B. Loret, \& N. Khalili (2012). A thermo-hydromechanical model in local thermal non-equilibrium for fractured HDR reservoirs with double porosity. Journal of Geophysical Research: Solid Earth (1978)-(2012) 117 (B7), DOI: 10.1029/2012JB009161.

Gelet, R., B. Loret, \& N. Khalili (2013). Thermal recovery from a fractured medium in local thermal non-equilibrium. International Journal for Numerical and Analytical Methods in Geomechanics 37(15), 2471-2501.

Gringarten, A., P. Witherspoon, \& Y. Ohnishi (1975). Theory of heat extraction from fractured hot dry rock. Journal of Geophysical Research 80, 1120-1124.

Hayashi, K., J. Willis-Richards, R. Hopkirk, \& Y. Niibori (1999). Numerical models of HDR geothermal reservoirs - a review of current thinking and progress. Geothermics 28, 507-518.

Khalili, N. \& B. Loret (2001). An elasto-plastic model for nonisothermal analysis of flow and deformation in unsaturated porous media formulation. International Journal of Solids and Structures 38, 8305-8330.

Khalili, N., S. Valliappan, \& C. Wan (1999). Consolidation of fissured clays. Géotechnique 49 (1), 75-89.

Kohl, T. \& R. Hopkirk (1995). "FRACture" - a simulation code for forced fluid flow and transport in fractured, porous rock. Geothermics 24, 333-343.

Loret, B. \& N. Khalili (2000). A three-phase model for unsaturated soils. International Journal for Numerical and Analytical Methods in Geomechanics 24, 893-927.

O’Sullivan, M., K. Pruess, \& M. Lippmann (2001). State of the art of geothermal reservoir simulation. Geothermics 30 (4), 395-429.

Taron, J., D. Elsworth, \& K.-B. Min (2009). Numerical simulation of thermal-hydrologic-mechanical-chemical processes in deformable, fractured porous media. International Journal of Rock Mechanics \& Mining Sciences 46, 842-854. 